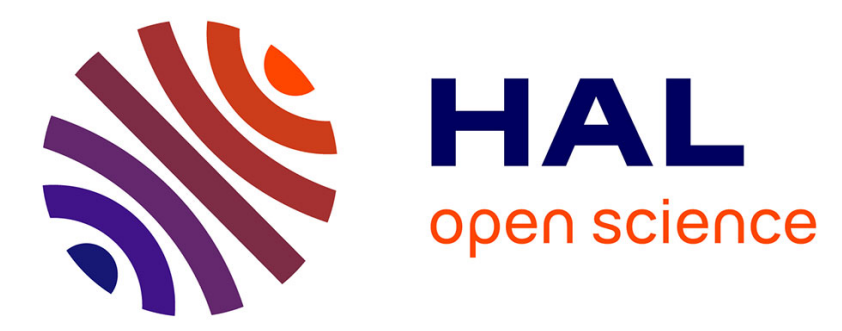

\title{
Role of buffer surface morphology and alloying effects on the properties of InAs nanostructures grown on $\operatorname{InP}(001)$
}

J. Brault, M. Gendry, G. Grenet, G. Hollinger, Y. Desieres, T. Benyattou

\section{To cite this version:}

J. Brault, M. Gendry, G. Grenet, G. Hollinger, Y. Desieres, et al.. Role of buffer surface morphology and alloying effects on the properties of InAs nanostructures grown on $\operatorname{InP}(001)$. Applied Physics Letters, 1998, 73 (20), pp.2932-2934. 10.1063/1.122634 . hal-02111653

\section{HAL Id: hal-02111653 \\ https://hal.science/hal-02111653}

Submitted on 26 Apr 2019

HAL is a multi-disciplinary open access archive for the deposit and dissemination of scientific research documents, whether they are published or not. The documents may come from teaching and research institutions in France or abroad, or from public or private research centers.
L'archive ouverte pluridisciplinaire HAL, est destinée au dépôt et à la diffusion de documents scientifiques de niveau recherche, publiés ou non, émanant des établissements d'enseignement et de recherche français ou étrangers, des laboratoires publics ou privés. 


\title{
Role of buffer surface morphology and alloying effects on the properties of InAs nanostructures grown on $\ln P(001)$
}

\author{
J. Brault, M. Gendry, G. Grenet, and G. Hollinger \\ Laboratoire d'Electronique-LEAME, UMR CNRS 5512, Ecole Centrale de Lyon, \\ 36 Avenue G. de Collongue, 69131 Ecully Cedex, France \\ Y. Desières and T. Benyattou \\ Laboratoire de Physique de la Matière-LPM, UMR CNRS 5511, INSA de Lyon, Bat. 502, \\ 69621 Villeurbanne Cedex, France
}

(Received 21 July 1998; accepted for publication 15 September 1998)

\begin{abstract}
We show the role played by the buffer surface morphology and by alloying effects on the size, shape and lateral distribution of InAs nanostructures grown on $\operatorname{InP}(001)$ substrates by molecular beam epitaxy. Three buffers, viz., $\operatorname{In}_{0.53} \mathrm{Ga}_{0.47} \mathrm{As}, \mathrm{In}_{0.52} \mathrm{Al}_{0.48} \mathrm{As}$, and InP lattice matched on InP have been studied. Differences in nanostructure morphology and in carrier confinement have been evaluated by atomic force microscopy and by low-temperature photoluminescence measurements, respectively. Alongside the classical relaxation mode through two-dimensional/three-dimensional surface morphology change, a chemical relaxation mode has to be introduced as a competitive mode of relaxation of strained layers. This chemical relaxation mode, due to alloying between the InAs deposit and the buffer, is thought to be responsible for most of the observed differences in the InAs nanostructure properties. () 1998 American Institute of Physics. [S0003-6951(98)03146-5]
\end{abstract}

Considerable work has been devoted to understanding and controlling the formation of self-organized quantum nanostructures resulting from the Stranski-Krastanov growth mode of III-V semiconductor strained layers. ${ }^{1}$ The real reason for such intense activity can be found in the potential application of quantum confinement in dots and wires usable in optoelectronic devices such as lasers or photodetectors. ${ }^{1,2}$ Highly strained systems like InAs/GaAs (lattice mismatch $\sim 7 \%$ ) have been extensively studied both experimentally ${ }^{3,4}$ and theoretically. ${ }^{5,6}$ However, quantum dot lasers in this system only operate at around $1 \mu \mathrm{m}$ while InAs quantum dots grown on InP could be promising for long wavelength lasers operating around $1.5 \mu \mathrm{m}$. Moreover, in such low strain systems (lattice mismatch $\sim 3 \%$ ), the self-organization process was thought to be less effective than for InAs/GaAs. Recently, a few reports have shown that InAs quantum dots can be grown on InP by metalorganic chemical vapor deposition (MOCVD) or molecular beam epitaxy (MBE). ${ }^{7}$ In addition, it appears that varying the matrix (InP, InGaAs, or InAlAs) could lead to different properties of quantum dots. ${ }^{8}$ In this letter, we compare the structural and electronic properties of InAs nanostructures grown by $\mathrm{MBE}$ on $\mathrm{In}_{0.53} \mathrm{Ga}_{0.47} \mathrm{As}$, $\mathrm{In}_{0.52} \mathrm{Al}_{0.48} \mathrm{As}$, and InP buffers lattice matched on InP. We explain the differences by variations in buffer surface morphology and alloying effects during growth of InAs. $\mathrm{In}_{0.53} \mathrm{G}_{0.47} \mathrm{As}$ and $\mathrm{In}_{0.52} \mathrm{Al}_{0.48} \mathrm{As}$ are analogous since they are quasibinary alloys with an InAs composition close to 0.5 , but they differ (i) by their growth front morphology: rougher for InAlAs than for InGaAs, and (ii) by their growth front chemical composition: InAlAs presents a larger InAs surface segregation than InGaAs for similar growth conditions. ${ }^{9}$ InP is a true binary and thus InAs/InP can be directly compared with the well known InAs/GaAs. The InAs deposit was fixed at a $3 \mathrm{ML}$ equivalent thickness just above the $2.5 \mathrm{ML}$ critical threshold for the two-dimensional/three dimensional (2D/
3D) growth mode transition measured by reflection highenergy electron diffraction (RHEED). Surface morphologies were characterized before (buffer surface morphology) and after (nanostructure morphology) the InAs growth using ex situ atomic force microscopy (AFM). In order to test the carrier confinement in these nanostructures, we have also performed photoluminescence (PL) at $4 \mathrm{~K}$ on the sample with InAs nanostructures embedded in an alloy or binary matrix similar to the buffer layer.

The samples were grown on (001) semi-insulating substrates by solid-source MBE in a Riber 2300 reactor. After thermal desorption of the native oxide on the $\operatorname{InP}(001)$ substrates, a $4000 \AA$ lattice matched buffer layer was grown. The growth conditions were chosen to be as close as possible to thermodynamic equilibrium. The growth temperature was fixed at $525{ }^{\circ} \mathrm{C}$ for the InAs/InGaAs and InAs/InAlAs and at $480{ }^{\circ} \mathrm{C}$ for InAs/InP in order to avoid any surface deterioration. The other growth conditions were the following: (i) InGaAs, InAlAs, and InP buffer layers were grown at a 1 $\mu \mathrm{m} / \mathrm{h}$ growth rate and a V/III beam equivalent pressure (BEP) ratio of 20, (ii) before the deposition of InAs a $10 \mathrm{~min}$ annealing step at growth temperature was made under As flux for InGaAs and InAlAs or under P flux for the InP, (iii) $3 \mathrm{ML}$ of InAs were grown at a reduced growth rate of 0.25 $\mu \mathrm{m} / \mathrm{h}(0.23 \mathrm{ML} / \mathrm{s})$. The arsenic pressure was fixed at $\sim 0.5 .10^{-5}$ Torr, as low as possible but compatible with Asstabilized surface growth, (iv) after InAs deposition, the samples were held $10 \mathrm{~s}$ at growth temperature. Then the samples were quickly cooled to $300{ }^{\circ} \mathrm{C}$ while arsenic pressure was maintained in order to reduce surface reorganization, (v) for samples designed for PL measurement, a $3000 \AA$ cap layer was added using the same growth conditions as for the buffer layer.

An AFM study was carried out to investigate the surface morphology on uncapped InAs nanostructures using a Park 

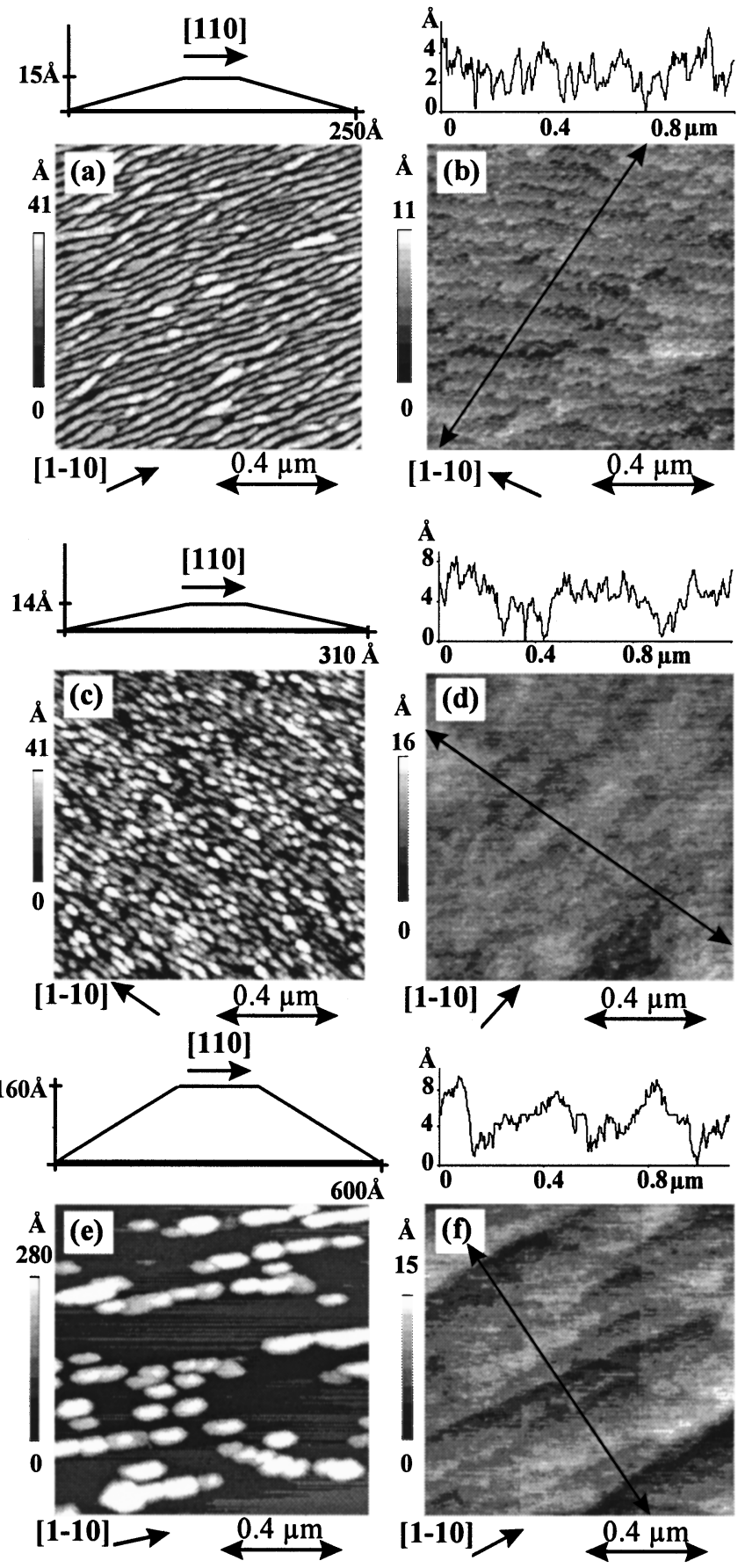

FIG. 1. Typical ex situ AFM images of the surface morphologies before (buffer surface morphology) and after (nanostructure morphology) the InAs growth: (a) InAs/InGaAs/InP, (b) InGaAs/InP, (c) InAs/InAlAs/InP, (d) InAlAs/InP, (e) InAs/InP/InP, and (f) InP/InP.

Scientific Instruments microscope, model CP, operating in the contact mode. In Fig. 1, typical InAs/InGaAs, InAs/ InAlAs, and InAs/InP images are shown and compared with those of the corresponding initial buffer. The buffer InGaAs and InP surfaces [Figs. 1(b) and 1(f)] are made of large, smooth terraces elongated along the $[1 \overline{1} 0]$ direction. Double steps can be seen on the InP buffer surface [Fig. 1(f)]. The InAlAs surface [Fig. 1(d)] is rougher with less distinct growth anisotropy along the [1 10$]$ direction. The shape, size, and lateral distribution of the nanostructures [Figs. 1(a), 1(c), and 1(e)] are quite different depending on the nature of buffer: (i) for InAs/InGaAs, 3D nanostructures appear as

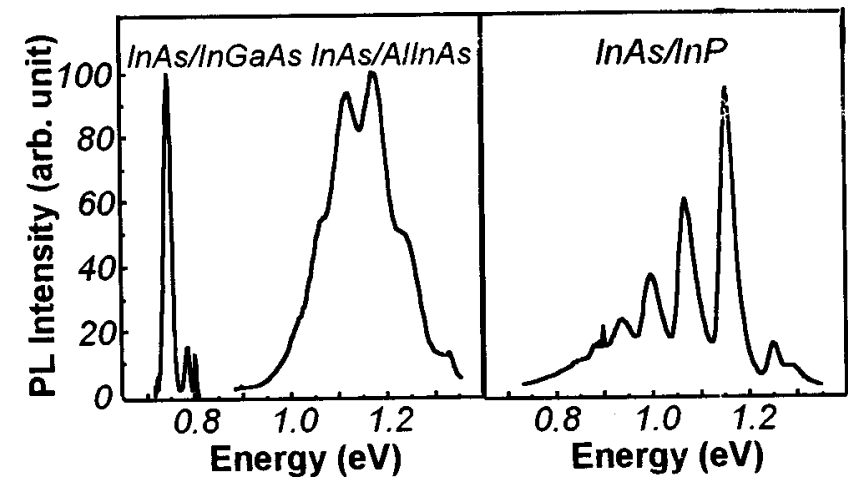

FIG. 2. Photoluminescence spectra at $4 \mathrm{~K}$ of InAs nanostructures embedded in InGaAs, InAlAs, and InP matrices.

closely joined wires of a few thousand $\AA$ long, $250 \AA$ wide and $15 \AA$ high elongated in the [1 10 ] direction, (ii) for InAs/ InAlAs, small and closely joined boxes of around $550 \AA$ long, $300 \AA$ wide, and $15 \AA$ high are distributed like nodes along the [1 $1 \overline{1} 0]$ direction, and (iii) for InAs/InP, dots appear as large and well-separated boxes, whose approximate dimensions are $1100 \AA$ long, $600 \AA$ wide, and $160 \AA$ high aligned along the $[1 \overline{1} 0]$ direction. The dot-dot distance varies from a few hundred $\AA$ in the $[1 \overline{1} 0]$ direction up to a few thousand $\AA$ in the [110] direction for the InAs/InP while for the other two systems it is comparable to the dot width. Large differences are also found in nanostructure area density which varies from $\sim 5 \times 10^{9} \mathrm{dots} / \mathrm{cm}^{2}$ for InAs/InP up to $\sim 8 \times 10^{10} \mathrm{dots} / \mathrm{cm}^{2}$ for InAs/InAlAs. For InAs/InGaAs, the area density of the nanostructures is approximately 10-20 times less than for InAs/InAlAs. Finally, when comparing the amount of deposited InAs with the total nanostructure volume, an excess of material was found in favor of the nanostructures for the InAs/InP (ratio 2.5), and to a lesser extent, for the InAs/InGaAs (ratio 1.3), but not for the InAs/InAlAs (ratio $\sim 0.6-1$ ). In other words, these results suggest that (i) all $3 \mathrm{ML}$ of InAs plus $4-5 \mathrm{ML}$ of the buffer layer form the dots in the InAs/InP, (ii) all $3 \mathrm{ML}$ of InAs plus $1 \mathrm{ML}$ from the buffer are used in the InAs/InGaAs to form the wires, and (iii) only about $2 \mathrm{ML}$ of InAs are used in the InAs/InAlAs to form the dots.

PL spectra are reported in Fig. 2. The main emission peak is located around $1.17 \mathrm{eV}$ for InAs/InAlAs and for InAs/InP, and at $0.74 \mathrm{eV}$ for the InAs/InGaAs system. A multiline photoluminescence spectrum appears for the InAs/ InP system. We have checked that this shape is not related to any interference effects within the structure. It has been previously proposed that this peculiar shape is related to a $1 \mathrm{ML}$ thickness modulation effect. ${ }^{10}$ Results on the influence of the excitation power confirm this attribution. A multiline spectrum is also obtained for the InAs/InAlAs system. The same PL studies as those carried out on the previous sample show that the PL shape originates from the quantum dot excited states, like those observed by Mukai et al. ${ }^{11}$ This shows efficient quantum confinement within the dots. A single narrow peak is detected for InAs/InGaAs. This is due to a lack of confinement because, in this case, the distance between wires is too small when compared to the small electronic gap difference between InAs and InGaAs. This is in good agreeto AIP license or copyright, see http://ojps.aip.org/aplo/aplcr.jsp 
ment with recently published results ${ }^{8}$ for $\operatorname{InAs} / \mathrm{In}_{0.52} \mathrm{Al}_{0.48} \mathrm{As}$ and $\mathrm{InAs} / \mathrm{In}_{0.53} \mathrm{Ga}_{0.47}$ As systems.

We attribute the observed drastic modifications in the nanostructure size with the nature of the buffer to competition between two simultaneous ways of relaxing the InAs overlayer/substrate mismatch strain. The composite formed by the buffer plus the strained overlayer can relax its excess energy via the well known 2D/3D surface morphology change and/or via some chemical exchange of the III or V elements in their respective sublattices. This latter phenomenon has already been theoretically proposed ${ }^{12-15}$ and evidenced experimentally in the case of InAs/GaAs for which a surface InGaAs alloy layer has been found for very small InAs deposits. ${ }^{16}$ For such weakly strained III-V systems such as InAs/InP, the chemical relaxation mode can become nonnegligible and thus must be introduced into the classical surface/bulk energy balance model. The key point for this second relaxation mode is the III-V alloy chemical instability due to a positive enthalpy of mixing $\Delta \mathrm{H}_{m}=x(1-x) \Omega$, where the interaction parameter $\Omega$ is a constant reflecting the energy excess associated with $\mathrm{AB}$ and $\mathrm{AC}$ bond distortion in the pseudobinary alloy $(\mathrm{AB})_{x}(\mathrm{AC})_{1-x}$. When $\Delta \mathrm{H}_{m}$ is small enough (small $\Omega$ or small $x$ ), the system can relax part of its total energy by producing an interfacial alloy between the overlayer and the buffer prior to any change of the growth mode. When comparing ${ }^{17}$ the interaction parameter $\Omega$ for a InAsP alloy $(\Omega \sim 720 \mathrm{cal} / \mathrm{mol})$, a InGaAs alloy $(\Omega$ $\sim 2490 \mathrm{cal} / \mathrm{mol})$ and a InAlAs alloy $(\Omega \sim 3600 \mathrm{cal} / \mathrm{mol})$, such overlayer/buffer alloying appears much easier for the InAs/InP [leading to $(\operatorname{InAs})_{x}(\mathrm{InP})_{1-x}$ nanostructures] than for the InAs/InGaAs [leading to (InAs) ${ }_{x}(\mathrm{GaAs})_{1-x}$ nanostructures] and for the InAs/InAlAs [leading to $(\mathrm{InAs})_{x}(\mathrm{AlAs})_{1-x}$ nanostructures]. In addition, in the first $2 \mathrm{D}$ growth stages of InAs on the InP buffer, As atoms can readily be included in the InP matrix because $x$ is small and thus $\Delta \mathrm{H}_{m}$ is small. On the other hand, for InAs/InGaAs and InAs/InAlAs, $x$ corresponds more or less to that of the mixing enthalpy $\Delta \mathrm{H}_{m}$ maximum $(x=0.5)$. We think that this is the main reason why the largest nanostructures are observed in the InAs/InP. The easy alloying of the deposited InAs overlayer with the buffer during growth leads to a larger number of monolayers being involved in the process. The resulting new InAsP phase induces less strain than InAs would have done.

Differences observed between InAs/InGaAs and InAs/ InAlAs are mainly associated with the role played by In surface segregation on the front growth of the two alloy buffers. It implies, first, an enrichment by one of the binaries at the surface, and, second, an increase in surface roughness to relieve the subsequent mismatch strain between surface and bulk layers. Because only a few surface top layers are concerned by a possible chemical exchange during MBE growth, the great intrinsic surface InAs enrichment of the InAlAs buffer will go against alloying with the InAs deposit. On the other hand, the InGaAs buffer presents less In surface segregation than $\mathrm{InAlAs}^{9}$ and thus deposit/buffer alloying can be more easily performed. The intrinsic surface roughness due to surface segregation (higher for the InAlAs buffer than for InGaAs) will favor nucleation because of the sites thus provided. This can explain the higher density and the

Downloaded 04 Feb 2003 to 156.18.34.206. Redistribution subject small size of the nanostructures in the InAs/InAlAs when compared to the InAs/InGaAs. Finally, for InAs/InGaAs and to a lesser extent for InAs/InAlAs, note that the nanostructure anisotropy [Figs. 1(a) and 1(c)] reflects the greater adatom surface diffusion along the [1 $1 \overline{1} 0]$ direction than along the [110] direction which gives the typical anisotropic surface morphologies for As-stabilized surfaces. ${ }^{18}$ In the InAs/ InP system, the nanostructure alignment is typical of the propensity for nucleation on predisposed sites of the buffer as a single or double step. ${ }^{19}$

We conclude from this study that the chemical and structural surface properties of the buffer can strongly influence the growth of low mismatched InAs nanostructures on InP. Alloying between the InAs deposit and the buffer is thought to be responsible for most of the observed differences in nanostructure size, shape, and distribution. The smaller the relevant mixing enthalpy is, the greater the alloying will be and so, the number of monolayers involved in the nanostructures. Furthermore, strong In surface segregation in the buffer can reduce this InAs overlayer/buffer mixing and produce an increase of the roughness responsible for the nucleation sites. These preliminary results clearly demonstrate the possibility of controlling the quantum dot shape, size, and distribution, and thus the carrier confinement, by varying the buffer composition.

The authors are grateful to P. E. Mazeran for introducing them to AFM imaging. This work is supported by the Region Rhoue-Alpes.

${ }^{1}$ For a review, see D. Bimberg, M. Grundmann, and N. N. Ledentsov, MRS Bull. 23, 31 (1998).

${ }^{2}$ P. M. Petroff and G. Medeiros-Ribeiro, MRS Bull. 21, 50 (1996).

${ }^{3}$ D. Leonard, K. Pond, and P. M. Petroff, Phys. Rev. B 50, 11687 (1994).

${ }^{4}$ J. M. Moison, F. Houzay, F. Barthe, L. Leprince, E. Andre, and O. Vatel, Appl. Phys. Lett. 64, 196 (1994).

${ }^{5}$ C. Priester and M. Lannoo, Phys. Rev. Lett. 75, 93 (1995).

${ }^{6}$ A. Madhukar, J. Cryst. Growth 163, 149 (1996).

${ }^{7}$ A. Ponchet, A. Le Corre, H. L'Haridon, B. Lambert, and S. Salaün, Appl. Phys. Lett. 67, 1850 (1995)

${ }^{8}$ V. M. Ustimov, E. R. Weber, S. Ruvimov, Z. Liliental-Weber, A. E. Zhukov, A. Yu. Egorov, A. R. Kovsh, A. F. Tsatsul'nikov, and P. S. Kop'ev, Appl. Phys. Lett. 72, 362 (1998).

${ }^{9}$ G. Grenet, E. Bergignat, M. Gendry, M. Lapeyrade, and G. Hollinger, Surf. Sci. 352, 734 (1996).

${ }^{10}$ K. Mukai, N. Ohtsuka, H. Shoji, and M. Sugara, Appl. Phys. Lett. 68, 3013 (1996); K. Mukai, N. Ohtsuka, M. Sugawara, and S. Yamazaki, Jpn. J. Appl. Phys., Part 2 33, L1710 (1994).

${ }^{11}$ J. F. Carlin, R. Houdré, A. Rudra, and M. Ilegems, Appl. Phys. Lett. 59, 3018 (1991)

${ }^{12}$ J. E. Guyer and P. W. Voorhees, Phys. Rev. Lett. 74, 4031 (1995); Phys. Rev. B 54, 11710 (1996).

${ }^{13}$ J. Tersoff, Phys. Rev. Lett. 74, 434 (1995); 77, 2017 (1996); Phys. Rev. B 56, R4394 (1997).

${ }^{14}$ F. Glas, Phys. Rev. B 55, 11277 (1997).

${ }^{15}$ F. Leonard and R. C. Desai, Phys. Rev. B 57, 4805 (1998).

${ }^{16}$ B. A. Joyce, J. L. Sudijono, J. G. Belk, H. Yamaguchi, X. M. Zhang, H. T. Dobbs, A. Zangwill, D. D. Vvedenski, and T. S. Jones, Jpn. J. Appl. Phys., Part 1 36, 4111 (1997).

${ }^{17}$ J. L. Martins and A. Zunger, Phys. Rev. B 30, 6217 (1984).

${ }^{18}$ J. Sudijono, M. D. Johnson, C. W. Snyder, M. B. Elowitz, and B. G. Orr, Phys. Rev. Lett. 69, 2811 (1992).

${ }^{19}$ R. Leon, T. J. Senden, Y. Kim, C. Jagadish, and A. Clark, Phys. Rev. Lett. 78, 4942 (1997).

AIP license or copyright, see http://ojps.aip.org/aplo/aplcr.jsp 